\title{
La formación de la ciudadanía desde la experiencia escolar
}

\author{
Yolanda Rodríguez \\ Pontificia Universidad Católica del Perú
}

\section{Rocío Domínguez}

Tecnológico de Monterrey, Campus Guadalajara

Yolanda Rodríguez es Socióloga y M.A. en Ciencias Políticas por la FLACSO; realizó estudios de post grado en Políticas Educativas en la Universidad Alberto Hurtado de Chile y actualmente cursa estudios doctorales en Educación. Es profesora del Departamento de Comunicaciones de la PUCP y miembro de la Sociedad de Investigación Educativa Peruana. Su área de investigación son las políticas educativas.

Rocío Domínguez obtuvo su doctorado en Adquisición de una Segunda Lengua de Carnegie Mellon University, en 2002. Ha trabajado como consultora en evaluación de proyectos educativos. Actualmente es profesora del Departamento de Idiomas del Tecnológico de Monterrey. Sus intereses son la introducción de innovaciones educativas y la capacitación de profesores en métodos de investigación cualitativa y en metodología de enseñanza de segundas lenguas.

El presente artículo se basa en la investigación del Instituto de Ética y Desarrollo (IED) de la Universidad Antonio Ruiz de Montoya (UARM) en la que participó una de las autoras. Esta se realizó en ocho colegios públicos y particulares de la red de instituciones educativas de la Compañía de Jesús en el Perú; a saber, colegios de la Red Fe y Alegría y colegios particulares jesuitas localizados en cuatro ciudades: Arequipa, Tacna, Lima y Piura. En dicho estudio realizado en 2007, se aplicaron, entre otros instrumentos, entrevistas semi-estructuradas a autoridades escolares y entrevistas grupales a estudiantes de tercero a quinto de secundaria. Las primeras secciones del presente artículo constituyen parte del análisis de contenido en la referida investigación -publicada recientemente por el Fondo Editorial de la UARM-, mientras que las referidas al análisis lingüístico fueron elaboradas posteriormente con ocasión del I Seminario de Investigación Educativa. El análisis lingüístico se aplica a una muestra de las entrevistas realizadas a los estudiantes en el año 2007 . El Seminario nos dio así la oportunidad de un diálogo interdisciplinar que contribuyó grandemente para una mejor comprensión del tema de estudio. Agradecemos al IED de la UARM por permitirnos utilizar el material de la investigación realizada por ellos. 


\section{La formación de la ciudadanía desde la experiencia escolar}

\section{Resumen}

El propósito del nuestro estudio es explorar las percepciones de estudiantes de secundaria acerca de ciudadanía y democracia, a partir de su experiencia escolar, comparando la producción de sus discursos ubicándolos en sus respectivos espacios escolares. Combinamos dos métodos: el análisis lingüístico, basado en la gramática sistémico-funcional, aplicado a las transcripciones de las entrevistas a estudiantes de tercero a quinto de secundaria de colegios particulares religiosos y públicos de la red de Fe y Alegría, ubicados en las ciudades de Arequipa, Lima, Piura y Tacna. El otro método comparó la información proveniente de documentos institucionales, entrevistas grupales a estudiantes y a autoridades escolares. Ambas líneas de análisis coinciden en señalar una participación de las organizaciones escolares más bien simbólica en la vida estudiantil.

Palabras clave: Ciudadanía, democracia, análisis del discurso, gramática-funcional, escuela.

\section{The development of citizenry from the school experience}

\section{Summary}

The purpose of our study is to explore on high school students' discourses about citizenship and democracy comparing their perceptions on both concepts with respect to their respective social contexts. Two independent methods were used for analyzing the data. We applied a linguistic analysis, based on the systemicfunctional linguistic theory, to the transcriptions of the interviews to students in grades 9th and 11th attending both catholic private schools and state schools that belong to the Fe y Alegría organization in the cities of Arequipa, Lima, Piura, and Tacna. Also, a thematic analysis compared portions of data coming from the schools' curriculum to data gathered by interviewing teachers, administrators, and students. Both lines of analysis point to similar findings showing a rather symbolic participation from student organizations in student affairs.

Keywords: Citizenship, Democracy, Discourse analysis, Functional grammar, School. 


\section{Introducción}

Es ampliamente aceptado que uno de los propósitos más importantes de la Eeducación escolar es la formación de ciudadanos; también lo es el papel que desempeña la escuela como instancia de socialización de niños y adolescentes como futuros ciudadanos. En la región latinoamericana el desarrollo de la identidad social y ciudadanía de los jóvenes es una preocupación relativamente reciente y creciente (Reimers \& Villegas-Reimers, 2006). ¿Qué hace la institución escolar para la formación de la ciudadanía? ¿Cómo está formando valores y actitudes para una ciudadanía democrática? ¿Cuál es su contribución a la tarea de formar ciudadanos democráticos?

Buscando responder a estas preguntas, en el año 2007 la Universidad Antonio Ruiz de Montoya realizó una investigación en los colegios de la red educativa de la Compañía de Jesús en el Perú. Los colegios son de gestión pública en el caso de la Red Fe y Alegría. Para el presente artículo trabajamos sobre el material recogido en el curso de esa investigación con el propósito de complementar los hallazgos del referido estudio, enfocándonos en los discursos de los estudiantes acerca de sí mismos como ciudadanos en formación. Nos proponemos explorar las ideas y percepciones de estudiantes de secundaria acerca de qué es ser ciudadanos y qué es la democracia, a partir de su experiencia escolar; identificar coincidencias y diferencias en la producción de los discursos de escolares sobre la ciudadanía y la democracia, ubicando estos en sus respectivos espacios escolares.

\section{Consideraciones conceptuales}

\section{Democracia, ciudadanía y el papel de la institución escolar ${ }^{1}$}

El concepto de ciudadanía refiere al ámbito de la política y está estrechamente ligado a la configuración de los Estados modernos, en donde la ciudadanía es la pertenencia a una comunidad de iguales, el Estado-nación, fuente de la soberanía del Estado. La pertenencia a la comunidad de iguales implica el reconocimiento de una serie de derechos y deberes de cada sujeto, el ciudadano. Como bien apunta López (1977), se trata de pasar de una ciudadanía imaginaria 
-limitada por factores de orden cultural, como institucional y político-, a una ciudadanía real, a una verdadera comunidad de ciudadanos con los mismos deberes y derechos efectivos. La construcción de la ciudadanía está así estrechamente vinculada con las posibilidades de integración e inclusión social de individuos y grupos que se ven limitados en el ejercicio de sus derechos y por tanto excluidos de la comunidad de ciudadanos.

La teoría política distingue entre diferentes tipos de democracia (Máiz, 2003): representativa, participativa, deliberativa e inclusiva; correspondiendo estas tres últimas a formas que toma la democracia en donde hay mayor espacio para la participación de la sociedad civil con el objetivo de garantizar la inclusión social. Aunque tienen diferencias importantes, principalmente en las formas de participación que propone cada una, en este artículo vamos a referirnos a la democracia participativa entendiéndola como participación para la inclusión social.

El acento en la participación, a diferencia de la democracia representativa que pone el énfasis en el ejercicio de elección de representantes, está justificado por la necesidad de garantizar la inclusión de los grupos denominados minoritarios históricamente excluidos del espacio político. De este modo, la democracia participativa establece como la principal característica de la democracia no el triunfo de las mayorías sino la posibilidad de los diferentes grupos de hacer llegar sus opiniones a los foros de decisiones políticas. Para Lüchmann (2006) la participación enfatiza la autodeterminación y la capacidad de transformación como factores centrales de la ciudadanía.

Aquí la participación se comprende como un tipo de participación política emergente que no es ni electoral ni de protesta; va más allá del ejercicio del derecho de voto y de participaciones eventuales motivadas por protestas puntuales. Se trata de una participación permanente, reconocida como parte del propio cuerpo del sistema político democrático.

Si la ciudadanía, y en este caso la participación como característica fundamental de la ciudadanía, es una construcción social (Fernández, 2001), ésta debe ser enseñada y aprendida. Es necesario transmitir a los nuevos ciudadanos los contenidos y valores de la ciudadanía democrática; lo que implica transmitir herramientas para participar y generar espacios para que se dé esta participación. Aunque esta transmisión es responsabilidad de la sociedad en su conjunto, varios autores coinciden en señalar que la escuela tiene un papel central en este proceso. ${ }^{2}$ En efecto, es función de la escuela 
impartir conocimientos (del sistema político, de los derechos, de los sistemas de participación, entre otros); desarrollar habilidades (resolución de conflictos, comunicación, cooperación, liderazgo); e inculcar valores (responsabilidad, tolerancia, confianza, solidaridad, justicia, honestidad, respeto, derechos de todos, libertad y apoyo al sistema democrático), que permitan la participación democrática de los individuos en la sociedad (Sabatini, 1998; Epstein, 1998; Caijao, 1998; Benavides, Cueto \& Villarán, 1999). Debe además ser entendida como eficaz (una vez que los sujetos de la participación reconocen el valor y necesidad de su participación) y finalmente requiere de participación efectiva ${ }^{3}$.

La escuela enseña no solamente a través del currículo (Reimers, 2006), sino también a través del currículo oculto en las practicas pedagógicas de los docentes y también a través de los modelos de roles que maestros, directivos, administrativos y todo personal de la escuela transmite a los estudiantes. Trabajar en el aula contenidos y valores relacionados con democracia y ciudadanía no tendrá gran impacto en la formación de los estudiantes, si los docentes y funcionarios de la escuela trasmiten en sus interrelaciones y en las interrelaciones de estos con los estudiantes comportamientos arbitrarios y discriminatorios.

El proceso de formación de ciudadanos democráticos desde la escuela presenta tres características (Reimers, 2006): a) integralidad, pues moviliza todos los espacios de la escuela; b) acumulación, pues como todo aprendizaje ésta debe ser entendida como parte de un proceso acumulativo, donde todo lo aprendido facilitará o dificultará aprendizajes posteriores; y c) cotidianeidad, porque se basa en los ejemplos y ejercicios cotidianos y no en los discursos específicos. Se trata pues de un proceso complejo que va más allá de los contenidos y discursos intencionales de los docentes.

\section{Aproximación conceptual al análisis del discurso}

El nuestro es un estudio cualitativo enmarcado metodológicamente en la teoría conocida como análisis del discurso. Dentro de esta teoría, nos valemos de la teoría lingüística sistémico-funcional desarrollada por Michael Halliday $(1985,1994)$, la cual se ocupa de analizar el lenguaje en términos

3 Ver Sabatini (1998) y Benavides et al. (1999). 
de los usos que le damos y los significados que transmitimos con él. Así, el lenguaje se entiende como un proceso social que se manifiesta o concretiza en diferentes contextos sociales. El análisis gramatical es funcional en tanto que se ocupa en describir cómo se usa el lenguaje en relación con quién, cómo, por qué y con qué fines se usa. El análisis funcional identifica cómo la estructura gramatical se concretiza en significados sociales y cómo estos significados construyen diferentes contextos ${ }^{4}$. Por esto mismo es que puede ser una buena herramienta para analizar las particularidades lingüísticas de textos orales de cara a las intenciones comunicativas de los hablantes. Otros estudios en los que se aplica la teoría sistémico-funcional para el análisis de textos orales y escritos son Schleppegrell \& Achúgar (2003) y Schleppegrell, Achúgar \& Oteíza (2004).

Los elementos de la oración se explican en referencia con su función en el sistema lingüístico y en asociación con variables contextuales para mostrar cómo los contextos situacionales se manifiestan a través de opciones lingüísticas particulares. Es necesario revisar brevemente algunas nociones básicas de la teoría sistémico-funcional para poder explicar a continuación cómo analizaremos las percepciones de los estudiantes acerca de la formación en democracia y ciudadanía que reciben en el colegio.

A través de la noción de registro, Halliday (1978) alude a la configuración de recursos léxicos y gramaticales usados para transmitir significados. Así, el registro se define como un conjunto de significados que corresponde a una función particular del lenguaje junto con palabras y estructuras las cuales expresan estos significados. Por ejemplo, se puede hacer referencia al registro matemático cuando el lenguaje se usa con fines y propósitos relativos a explicar conceptos matemáticos. El registro no se limita en los recursos léxicos, sino que también involucra nuevos estilos de significar, formas de desarrollar una argumentación y nuevas maneras de combinar elementos ya existentes. Dado que el significado se construye a través del lenguaje, el registro es la manifestación lingüística de un contexto social del lenguaje.

De acuerdo con la gramática funcional, el registro varía por la interacción de tres variables denominadas campo, tenor y modo. Campo se refiere a las ideas contenidas en el texto; tenor es la relación que se establece entre el hablante-autor/oyente-lector; y modo son las expectativas de cómo los tipos

4 En la teoría sistémico-funcional, el término contexto alude a la situación misma en la que se produce el texto, sea este oral o escrito. 
de textos se organizan. Dos textos pueden referirse al mismo campo, pero pueden tener opciones léxico-gramaticales diferentes debido, por ejemplo, a que están dirigidos a públicos distintos (diferencias en el tenor); o a que están organizados de diferente manera (diferencias en el modo).

Siguiendo la teoría sistémico-funcional, las variables campo, tenor y modo se manifiestan a través de opciones léxico-gramaticales en cada registro construyendo tres metafunciones o formas de construir significados: la ideacional, la interpersonal y la textual.

La forma ideacional alude a la construcción de significados en relación con la presentación de las ideas en el texto que incluye a los participantes (quienes realizan la acción), los procesos y las circunstancias en las que estos procesos se dan. Además, las ideas presentadas en el texto tienen una conexión lógica.

En general, la mayoría de las teorías gramaticales se limita a estudiar los elementos de la forma ideacional. El aporte de la teoría sistémico funcional está en que además incorpora las otras dos dimensiones, la interpersonal y la textual en el análisis de los registros.

La forma interpersonal se refiere a la manera en la que se da la relación entre hablante-autor/oyente-lector. Las opciones gramaticales a través de las cuales se manifiesta esta relación incluyen el uso de preguntas, afirmaciones, órdenes o a través del uso de verbos modales, la entonación y otros recursos lingüísticos que reflejan opinión y actitud. Los siguientes ejemplos ilustran cómo se manifiesta el tenor:

(1) J: ...y entonces no pudimos abrir la puerta...

P: ¿por qué? ¿estaba con llave?

J: no, no estaba con llave, pero estaba atascada...

(2) El uso de cinturones de seguridad contribuye a reducir el número de muertes en los accidentes de tránsito.

(3) Tome con moderación.

En el ejemplo 1, ambos participantes contribuyen a crear el significado del texto. En el ejemplo 2 se afirma indirectamente una posición a favor del uso de los cinturones de seguridad. Por último, en el ejemplo 3 se da una orden.

Finalmente, la forma textual hace referencia a la forma en la que se estructura el texto de acuerdo con la función que cumple en una situación determinada. La estructura de un texto es diferente si este constituye una ponencia o si es el producto de una actividad social como en una reunión de trabajo o si el lenguaje va acompañado de recursos visuales como cuando se presentan 
diapositivas. La forma textual alude también a la distancia y la disponibilidad de retroalimentación entre el hablante-autor/oyente-lector. En las interacciones orales, por lo general, los participantes co-construyen el significado de un texto como se muestra en el ejemplo 1.

En resumen, la noción de funcionalidad implicada en la teoría sistémicofuncional alude a estas metafunciones en el sentido en que en cada cláusula del español ocurren simultáneamente tres procesos: se habla de algo, se establecen relaciones sociales y se estructura un texto. Por lo anterior se puede hablar de un análisis lingüístico multifuncional.

\section{La noción de metáfora gramatical}

Para poder analizar la naturaleza de los registros académicos en comparación con el registro del lenguaje del día a día, Halliday (1994) se vale de la noción de metáfora gramatical. Esta noción se refiere al uso singular de categorías gramaticales para transmitir significados. En el lenguaje cotidiano no se usan metáforas gramaticales, más bien se favorece el uso de expresiones congruentes en las cuales los objetos y las acciones se manifiestan lingüísticamente por medio de sustantivos y verbos, respectivamente. Las circunstancias se expresan a través de adverbios y frases preposicionales y las relaciones entre los elementos se dan por medio del uso de conectores. En cambio, el lenguaje del registro académico no sigue esta correspondencia. A través del uso de metáforas gramaticales se expresan conceptos en forma "compacta", mientras que el lenguaje cotidiano favorece la perífrasis. Asimismo, el lenguaje académico está lleno de términos técnicos y especializados.

Para concluir, en nuestro estudio nos valdremos de las nociones de la gramática funcional antes mencionadas para analizar lingüísticamente las percepciones de los estudiantes acerca de la formación ciudadana y en democracia del colegio en donde cursan estudios.

\section{Metodología}

En el estudio se combinaron dos métodos. Uno de ellos consistió en aplicar un análisis lingüístico, basado en la gramática funcional, a las transcripciones 98 I de las entrevistas grupales a estudiantes de tercero a quinto de secundaria de 
colegios particulares religiosos y públicos de la red de Fe y Alegría, de cuatro ciudades: Arequipa, Lima, Piura y Tacna. El otro método comparó la información proveniente de: a) documentos institucionales en los que los colegios declaran los objetivos educativos, b) las entrevistas grupales a estudiantes, y c) entrevistas individuales de autoridades escolares (directores, profesores y tutores) $)^{5}$.

En las entrevistas a las autoridades escolares se aplicó un análisis de contenido sobre los objetivos del colegio con relación a la formación de la ciudadanía y los valores democráticos, así como las formas de su implementación en la currícula. El análisis de los documentos institucionales de los colegios se enfocó en los objetivos educativos y su relación con la formación de la ciudadanía y valores democráticos.

\section{Sobre el análisis lingüístico}

Como parte del acopio cualitativo de información, se grabaron entrevistas grupales a estudiantes de ocho colegios. Estas fueron luego transcritas textualmente.

Usando las herramientas proporcionadas por la gramática funcional, se aplicó un análisis lingüístico a las transcripciones. Este consistió en contrastar al interior de cada transcripción las partes en las que los alumnos comparten sus percepciones sobre la formación en valores democráticos y ciudadanos, con aquellas partes en las que narran los planes de trabajo de las organizaciones estudiantiles (consejo escolar o municipio escolar, según el colegio), las actividades y su participación en la vida escolar. La unidad de análisis es la cláusula de acuerdo con la definición de la teoría sistémico-funcional, es decir, la unidad mayor con sentido para el análisis gramatical.

De esa manera, una contribución de este estudio es usar dos líneas de análisis independientes aplicadas a los datos recogidos, complementándose y reforzando los hallazgos. 


\section{El contexto: la ciudadanía y los valores democráticos en las propuestas educativas de los colegios}

Tanto los colegios de la red Fe y Alegría como los colegios particulares jesuitas tienen referencias explícitas a elementos relacionados con la formación de la ciudadanía en sus proyectos educativos. Más aún, estos elementos constituyen ejes que dan identidad y sentido a la educación que ofrecen a la sociedad; una educación pensada para la formación de personas capaces de participar -libre, solidaria y eficazmente- en la construcción de una sociedad democrática. Las instituciones educativas "educan desarrollando una ética que respeta la dignidad de cada uno y hace posible la libertad, la convivencia democrática y pacífica y el respeto a los derechos humanos" (Conferencia de Provinciales Jesuitas de América Latina, 2005, p. 6).

Así, la formación integral que ofrecen los colegios jesuitas se traduce en educar personas capaces de colaborar en la construcción de una sociedad justa y solidaria:

... una comunidad sin exclusiones basada en el respeto a la persona, a su identidad cultural y a su libertad; que asegura las condiciones materiales que brindan a todos las posibilidades de desarrollo humano. Se trata de una sociedad donde se respeta la diversidad de raza, sexo, lengua, cultura y religión. Una sociedad de ciudadanos que participan de la cosa común como miembros responsables de una colectividad mayor. (Compañía de Jesús 2002, pag.17)

Mientras que en la Propuesta Educativa de Fe y Alegría, la educación integral de las personas implica un compromiso institucional con la generación de

... una cultura de paz, que ayude al desarrollo de una ciudadanía responsable, que construya democracia, participación organizada e institucionalidad, desde la práctica; consciente y responsable también de su tarea de conservar, transmitir y generar cultura, con valoración y estima de lo propio, estima y respeto de las diferencias, vocación por la fe, la justicia y la solidaridad... (Fe y Alegría 2005, p. 6)

Esta orientación se concreta en los proyectos pedagógicos a través del 100 I desarrollo de capacidades, habilidades, actitudes y valores; así como en la 
contextualización de los aprendizajes, la selección de los contenidos relevantes y pertinentes del entorno para que los alumnos conozcan, interpreten y actúen en su realidad. La institución escolar tiene una importancia destacada como espacio de reflexión e interpretación permanente de la realidad; es el lugar donde los jóvenes se preparan para actuar en ella, asumiendo responsabilidades y compromisos para transformarla (Compañía de Jesús, 2002).

Las capacidades, habilidades, actitudes y valores que buscan desarrollar en los educandos tienen que ver con disposiciones propias de una ciudadanía democrática: la búsqueda del diálogo y conocimiento de los otros mundos sociales (especialmente las realidades sociales de la pobreza); la capacidad de encarar los conflictos sociales y brindar los medios para la búsqueda de consensos (Compañía de Jesús, 2002, p. 20); sensibilidad social para descubrir su relación con los demás y con el entorno, desterrando todo tipo de exclusión y discriminación. Los medios por los que los colegios procuran lograr estos propósitos son el fomento de la participación activa de los estudiantes en la vida escolar; el trabajo en equipo; la aplicación de métodos activos y uso de diversas estrategias metodológicas y medios; el desarrollo de la reflexión y el sentido crítico; la capacidad de iniciativa, de organización y creatividad; el ejercicio de la libertad y la toma de decisiones.

Como fruto de esta formación integral, se espera que al término de su educación los alumnos de los colegios jesuitas presenten un conjunto de características (perfil) en las dimensiones religiosa, afectiva, intelectual, psicomotriz, social y ética. Con relación a esta última dimensión, el perfil es que el alumno sienta como propios los efectos de un orden social injusto e inhumano y comprenda que es responsable en la búsqueda de soluciones; sea capaz de comprometerse solidariamente, especialmente con los más pobres, con quienes reconozca tener un compromiso especial; ame a su patria, conozca sus problemas sociales y las alternativas de desarrollo que se plantean desde las distintas corrientes ideológicas o partidarias y esté en capacidad de asumir una posición personal; promueva una auténtica democracia que favorezca el desarrollo personal y comunitario de la nación; conozca y haga respetar sus derechos y sea fiel en el cumplimiento de sus deberes; reconozca los límites que imponen al uso de su libertad los derechos de los demás y actúe en consecuencia; rechace el racismo, el machismo y toda forma de marginación y discriminación social de acuerdo con su vocación a la fraternidad y su conciencia de la dignidad de la persona humana y promueva una cultura de paz, interculturalidad y equidad 
de género; limite razonablemente, y por propia iniciativa, comodidades y acciones que no son coherentes con la sobriedad y austeridad cristianas en medio de la pobreza que marca nuestra sociedad.

La Educación en Valores constituye uno de los tres pilares de la Propuesta Educativa de Fe y Alegría, junto con la Educación Popular y la Educación en y para el Trabajo. La Educación Popular vendría a expresar el objetivo institucional de entregar una educación de calidad a sectores empobrecidos, para la formación de líderes y ciudadanos orientados a la transformación social. La Educación Popular implica también la inserción de los colegios en las comunidades que los acogen, participando activamente en la dinámica de la vida local.

La educación en valores, el otro eje de la formación integral, se centra en el desarrollo de la conciencia moral que conduzca a la decisión autónoma y libre del y la joven de involucrarse activamente en la transformación de la sociedad.

Más que limitarse a transmitir valores, a Fe y Alegría le interesa propiciar que los miembros de sus comunidades educativas opten voluntariamente por ellos; que desarrollen actitudes de adhesión, nacidas de una profunda reflexión y sensibilidad personal, ya que ésta es la única garantía de que su actuar será consecuente y coherente, más allá de los muros y los tiempos de la escuela. (Fe y Alegría, 2005, p. 15)

La formación en valores debe expresarse en el proyecto educativo institucional (PEI) de cada colegio Fe y Alegría y concretarse en el diseño o planificación curricular de cada centro educativo. En efecto, los directivos y profesores entrevistados han destacado la centralidad de la formación en valores en la propuesta educativa de los colegios. Esta "atraviesa la propuesta curricular". Se elaboran "matrices de valores" que son particulares a cada colegio, pues están relacionadas con su problemática específica. Estos ejes transversales deben reflejarse en todos los cursos del currículo escolar, tanto de primaria como de secundaria, y no limitarse a los contenidos de los cursos del área de Ciencias Sociales. Formar en valores significa un conjunto de aspectos de orden tanto cognitivo, como afectivo y conductual, tales como la elección entre alternativas para tomar decisiones; el conocimiento de principios universales como los derechos humanos; el fortalecimiento de hábitos y de la voluntad; la capacidad de asumir los errores como forma de seguir aprendiendo; la conciencia y responsabilidad de los educadores de su rol de referentes.

La Propuesta Pedagógica de Fe y Alegría establece también cómo educar 102 I en valores, señalando un conjunto de dimensiones relativas a la calidad de las 
relaciones interpersonales (relaciones horizontales y de confianza mutua) y el clima institucional (diálogo); así como a las interacciones pedagógicas (discusión controversial, capacidad empática, reflexiva) y el tratamiento integral a los contenidos transversales en el proceso educativo, "para abrir espacios de análisis de la realidad y dar lugar a la participación organizada (consejos estudiantiles, delegados, comisiones, clubes, entre otros) en la solución y respuesta a problemas evidenciados, reflejando y afirmando los valores asumidos" (Fe y Alegría, 2005, p. 16).

Los aprendizajes que Fe y Alegría considera necesarios conducen a que

... el alumno esté en capacidad de desenvolverse con eficiencia, eficacia y satisfacción tanto en su vida personal y social como en su relación con el entorno natural, y que se comprometa a la acción transformadora de una sociedad mejor para todos. (Fe y Alegría, 2005, p. 19)

Uno de los aprendizajes fundamentales es la participación democrática; Fe y Alegría entiende la democracia como una cultura (modo de ser y de actuar), que se traduce en relaciones participativas, cooperativas y solidarias en todos los ámbitos de la vida. Su aprendizaje, por tanto, es práctico: "la acción educativa debe promover diversas formas de participación y organización, desarrollando la conciencia del bien común, el sentido de pertenencia y de corresponsabilidad con un proyecto comunitario" (Fe y Alegría, 2005, p. 35).

Estos elementos, que constituyen el marco pedagógico orientador de la labor educativa de los colegios de Fe y Alegría, se concretan en el proyecto curricular de cada centro educativo. El documento Propuesta Pedagógica señala que el diseño curricular debe ser fruto del análisis del contexto nacional y local (el entorno del centro educativo y la misma institución). Este análisis da lugar a los ejes curriculares y los contenidos transversales ${ }^{6}$. Uno de los ejes curriculares señalados en el documento es el desarrollo de una conciencia ética, democrática y ciudadana, que deberá traducirse en comportamientos responsables y solidarios, en participación consciente y organizada, en trabajo cooperativo y relaciones horizontales.

6 Los ejes curriculares son ideas directrices que expresan la intencionalidad educativa, a partir de las cuales se seleccionan los contenidos, habilidades, valores y actitudes que se desarrollarán en el currículo. Estos ejes son: identidad personal y cultural; cultura creadora y productiva; conciencia ética, democrática y ciudadana. 
Los contenidos transversales "reflejan las necesidades prioritarias de aprendizaje en torno a problemas que afectan la vida cotidiana de sus miembros [de la comunidad educativa]. Establecen el nexo entre la escuela y la vida misma" (Fe y Alegría, 2005, p. 60). Estos contenidos no se deben limitar a lo que se enseña en el currículo, sino que deben reflejarse en el conjunto de la vida institucional, sobre todo en las formas y estilos de relacionamiento entre los miembros de la comunidad educativa. La Propuesta Pedagógica plantea un conjunto de ocho contenidos transversales, los que responderían a la problemática común de los contextos en los cuales se ubican los colegios; compete a cada colegio seleccionar "uno o dos para darles tratamiento durante el año y proyectarlos a la comunidad" (Fe y Alegría, 2005, p. 60).

Los ocho contenidos transversales están relacionados con la formación de la ciudadanía: derechos del niño y del adolescente, conciencia ambiental y calidad de vida, interculturalidad, identidad personal y relaciones de género, trabajo, tecnología y productividad, ética y cultura de paz, ciudadanía y democracia, fe y justicia. Destacamos tres contenidos por su relación con el tema que nos ocupa: a) el contenido "interculturalidad" hace referencia al conocimiento y valoración de la diversidad cultural; b) el contenido "relaciones de género" se refiere al desarrollo de relaciones de género justas y equitativas; $y$ finalmente c) el contenido "ciudadanía y democracia" plantea la participación libre y responsable en la vida de la comunidad, en la vigilancia de los compromisos y en la toma de decisiones.

\section{Discusión}

\section{Aprendiendo a ser ciudadanos en la experiencia escolar}

Para las autoridades escolares y los profesores, el proyecto educativo de la institución tiene como uno de sus ejes centrales la formación de los estudiantes como futuros ciudadanos. Para ello disponen de una serie de recursos y de medios que deben permitir a los escolares tener experiencias en el cotidiano de la escuela a través de las cuales descubran valores, actitudes y comportamientos necesarios para una convivencia democrática. Se trata de una gama muy amplia que incluye la elaboración de las normas de convivencia en el aula, la participación en comisiones de trabajo, la elección de los representantes estudiantiles, actividades de proyección social; como también estilos de enseñanza que buscan la expresión 104 I libre de las ideas, el desarrollo de la capacidad de argumentación y debate, el 
discernimiento y la toma de decisiones; y la organización de los aprendizajes dando especial atención al descubrimiento de su realidad desarrollando en los estudiantes un sentido crítico y constructivo.

Profesores y alumnos coinciden en destacar entre estos medios, la organización y la participación de los estudiantes en la vida escolar a través de los municipios escolares y los consejos estudiantiles ${ }^{7}$. Estas son organizaciones de los estudiantes a nivel del centro escolar, reconocidas por las autoridades escolares, integradas por alumnos elegidos por sus compañeros, con el fin de representar y expresar sus intereses y canalizarlos a través de actividades libremente planteadas por ellos. Estas organizaciones son concebidas por las autoridades escolares como espacios formativos porque permiten la incorporación temprana y activa de los escolares en un proceso de desarrollo de valores, ciudadanía y democracia. Son espacios de aprendizaje en la medida en que promueven su entrenamiento en la práctica de métodos y actitudes democráticas; así como una mayor horizontalidad en las relaciones entre profesores y alumnos ${ }^{8}$.

Desde la institución educativa se trata de una concepción de la escuela como espacio privilegiado para la formación temprana de valores ciudadanos y democráticos. Desde la perspectiva de los estudiantes, la elección de sus representantes es la principal experiencia vivida en el colegio que contribuye a su formación como ciudadanos; esta experiencia les aporta un aprendizaje que más tarde van a poder aplicar como ciudadanos responsables del devenir de su comunidad, ejerciendo su derecho de elección con base en el análisis de las ideas y de los "líderes que mejor los van a llevar al progreso" (entrevista estudiante F).

El análisis de los planes de trabajo de las organizaciones estudiantiles y las entrevistas con estudiantes y profesores sugieren algunos desencuentros entre este ideal y sus realizaciones actuales ${ }^{9}$. Muchas veces las organizaciones

$7 \quad$ En los colegios de la Red Fe y Alegría estas organizaciones reciben el nombre de Municipio Escolar y están conformados por alcaldes y regidores; el municipio escolar tiene un concejo escolar, concejos de aula y comisiones de trabajo. En los colegios particulares estas suelen denominarse Consejo Estudiantil e interactúa con los delegados de aula con quienes debe tener un estrecho vínculo.

8 En Eguren (2006) se encuentra una interesante discusión sobre los enfoques y prácticas de promoción de la participación en la escuela desde el Estado, la sociedad civil y las escuelas. En otro estudio, Carbajo \& Espino (2001) analizan los contenidos de las propuestas de municipios escolares de Ayacucho y encuentran que estos privilegian la atención a carencias tangibles como el arreglo o ampliación de infraestructura escolar; los autores señalan que estos planes estarían en general absorbiendo actividades no cumplidas por los adultos, constituyendo respuestas a la carencia que estarían dejando "en segundo plano las urgencias propiamente juveniles" (p.17). 
estudiantiles funcionan como facilitadoras de las autoridades escolares cumpliendo un rol de apoyo a la vigilancia del orden, conservación de la limpieza y administración del castigo ${ }^{10}$.

Otras veces, las organizaciones cumplen un rol de correas transmisoras entre las autoridades escolares y sus representados facilitando la difusión de información que la institución escolar estima como de necesario conocimiento por el alumnado. Corresponderían así al nivel de participación simbólica o decorativa (Alfageme, 2003).

En otros contextos escolares hemos podido observar que existen en los colegios figuras mediadoras de los conflictos y aspiraciones de los estudiantes y los canales instituidos para procesar estos. Es el caso de los sacerdotes (llamados padres espirituales) con una relación muy cercana a los estudiantes que puede resolver muchas veces una situación de una manera más rápida que si ésta se procesara por los canales establecidos (delegados de aula, consejo estudiantil, profesores encargados de formación o de disciplina, por ejemplo). Esta figura tradicional a veces actúa como "colchón" limitando el ejercicio de la representación y el desarrollo de la capacidad de negociación de conflictos de las organizaciones estudiantiles.

Un elemento importante a considerar es el rol de los y las profesores tutores, quienes tienen un papel destacado en la orientación de las organizaciones de representación estudiantil, así como en el diseño y ejecución de su plan de trabajo anual. Más allá de ello, en los colegios existe la figura del tutor asociada con la formación integral de los estudiantes, no reducida a la dimensión académica. El rol de tutoría es central porque es desde donde, de manera más explícita, se establecen los puentes o nexos entre el proceso de aprendizaje y la inserción de éste en un contexto social e histórico particulares. La contextualización de los aprendizajes que permitan concretar la opción de una educación para formar personas comprometidas con su país.

Encontramos que en los colegios públicos el rol del tutor o tutora no está siendo suficientemente elaborado y reflexionado ${ }^{11}$. En general, los colegios públicos y particulares no capacitan a los profesores para la labor de tutoría y estos se sienten poco habilitados para responder a las demandas de esta función. No es frecuente encontrar que se "problematice" la función del tutor, que se construya como un tema de estudio y reflexión en los espacios

10 Esto está más presente en los colegios de la Red Fe y Alegría que en los colegios particulares.

11 Nos referimos a los tutores designados para asesorar a los municipios escolares o consejos estudiantiles. 
de conversación que la institución escolar reserva para el encuentro de los profesores.

No queremos reducirnos a una dimensión de capacitación del rol de tutoría; el asunto tiene más que ver con las visiones que tienen los profesores, las autoridades y que comparten muchas veces los estudiantes, acerca del sentido de la participación y de la organización estudiantil. Tal como un grupo de profesoras del área de Ciencias Sociales entre las que hay tutoras y asesoras, piensa que el rol del municipio escolar es la "participación en todo lo que necesite el colegio; es apoyo al trabajo de los profesores". (Entrevista profesoras F3). O de apoyo a los tutores:

Los Compañeros Guía ${ }^{12}$ ayudan a detectar problemas de conducta en sus compañeros, son los que ayudan a los tutores; ayudan a los auxiliares, pero ellos no sancionan sino derivan casos al tutor o al auxiliar. Cumplen funciones de control de ingreso al colegio, chequean que los alumnos parten sus agendas firmadas por los padres familia, que estén correctamente uniformados. .. es una labor de ayudantía. (Entrevista asesor F).

En síntesis, las organizaciones estudiantiles parecen correr el riesgo de estar expresando prácticas que se convierten en rutinarias y formales; dos razones pueden dar cuenta al menos parcialmente de ello. De un lado, lo poco problematizadas que son estas fórmulas y, de otro, el excesivo peso en las dimensiones de disciplina y control en desmedro de la participación y la expresión juvenil.

\section{El descubrimiento de la diferencia}

Como ha sido presentado anteriormente, este es un propósito fundamental en el proyecto formativo de los colegios; exploramos este punto desde la relación que establece la institución escolar con el entorno y las familias.

Los colegios aspiran a ser comunidades educativas insertas en el medio social. Esto es, no desean permanecer ajenos a sus realidades circundantes sino actuar dinámicamente con ellas. Ello se traduciría en el reconoci-

12 Los Compañeros Guía (CG) existen en los colegios de Fe y Alegría; son elegidos entre los estudiantes de mejor rendimiento académico a propuesta del tutor o tutora y es un cargo rotativo a lo largo del año escolar hay un comité de CG de primaria y otro de secundaria. 
miento del contexto; la incorporación de elementos de éste en la gestión institucional y curricular; la proyección a la comunidad buscando aportar a su desarrollo; y el conocimiento y relación estrecha con las familias de los estudiantes.

Cabe preguntarnos por la lectura de la realidad local que hace la institución escolar pues a partir de ella definen los ejes curriculares y los contenidos transversales de cada centro educativo. Los documentos de gestión analizados muestran que las referencias al contexto están por lo general anotadas como amenazas del entorno, casi nunca como oportunidades para el proyecto educativo. En el entorno se identifica la presencia de violencia, pandillas, desorganización y desunión de la comunidad, falta de liderazgo, delincuencia, drogadicción, internet, televisión, prostitución y alcoholismo. De otro lado, la lectura del entorno familiar de los alumnos destaca por lo general características que son vistas como amenazas para el proyecto educativo. La familia es vista casi siempre como problema -desintegración familiar, padres separados, abandono, desunión y violencia familiar, irresponsabilidad de los padres-; fuente de carencias que afectan el desenvolvimiento escolar: carencia de hábitos de estudio y de higiene, mala alimentación, agresividad, maltrato y baja autoestima.

De este diagnóstico genérico se concluye directamente, sin mayores mediaciones, que el problema es la carencia de afecto en el hogar y su efecto, las conductas violentas o agresivas de los escolares. Sin intentar negar esta posible asociación, observamos que existe un conocimiento limitado de la realidad familiar y social de los estudiantes por parte de la institución educativa. Prima una visión ideal de la familia que, al no estar presente como realidad en la mayoría de los casos, lleva a asociar a la familia "real" con rasgos negativos. Nos atreveríamos a decir que el estereotipo acerca de la familia y del entorno reemplaza muchas veces el conocimiento real de estos.

Los comportamientos no deseados, por agresivos o violentos, son traídos de "afuera" del colegio, del entorno:

Estos alumnos nuevos han traído consigo esas costumbres; [son] violentos, agresivos, porque vienen de hogares desintegrados, de familias donde hay mucha violencia. . . Los profesores trabajamos, pero ¿qué pasa en los hogares? Provienen de hogares desintegrados. Nuestros alumnitos están adoptando, imitando esas conductas, escriben malas cosas en el baño en contra de sus compañeras, en el recreo muestran actitudes violentas, se empujan. (Entrevista profesor F). 
Esta distancia del colegio con relación al medio que lo rodea, queda bien expresada en la siguiente imagen de una profesora de Ciencias Histórico Sociales que lleva diez años trabajando en el colegio:

Fuimos a limpiar el cerro, que no tiene agua ni nada. Yo nunca había subido; es curioso que nunca hubiera subido. Desde arriba se ve el colegio muy lejano, no está no siquiera cerca al cerro y los alumnos viven en el cerro. Subimos hasta la parte más alta y el olor era bien fuerte, demasiado fuerte . . en realidad estaba feo. Es muy pobre . . las esteritas . . cada casa no sé si tendrá silo. El hermano me preguntó: "¿Cómo has visto el colegio desde arriba?". Bueno, lo he visto lejano ... ., no tienen agua, no tienen luz, [no satisfacen] las necesidades básicas y necesitan todo. Yo veo al colegio como algo lejano, un pequeño espacio donde hay gente que se preocupa por los alumnos, pero solamente dentro del colegio. A mí sí me impactó. Yo sinceramente pienso que el colegio es un puntito chiquitito en comparación con todo lo que hay. (Entrevista profesora F).

Un descubrimiento que llama a atención es la práctica inexistencia de actividades de proyección social en los colegios públicos y su limitada implementación en los colegios particulares. Sólo en un caso hay una actividad de proyección social articulada con el desarrollo del currículo y de participación obligatoria para todos los estudiantes en el tránsito de un grado a otro de la secundaria. Así mismo, los colegios particulares suelen ser "islas" en su medio, no se ve una intencionalidad de integrarse activamente en su ámbito educativo local, interactuando con colegios públicos del ámbito ${ }^{13}$.

El encuentro con el "otro" que posibilitan los proyectos de proyección a la comunidad, cuando no tienen una carga meramente asistencialista, resultan una dimensión importante en la formación del sentido ciudadano. Colaboran significativamente con la ruptura de prejuicios muy acendrados en la cultura racista, la cual atribuye características negativas a las personas en razón de su diferente origen social o por sus características físicas ${ }^{14}$.

13 Un colegio particular tiene una proyección diferente, participando en los espacios de coordinación de instituciones educativas de su mismo ámbito y promoviendo actividades de intercambio entre los estudiantes del último año de secundaria; estas actividades están institucionalizadas en la cultura escolar desde hace muchos años.

14 Asimismo el estudio de Rodríguez (2009) halló estereotipos de género, tanto sobre los estudiantes como sobre los profesores. 
Por otra parte, las manifestaciones de discriminación en el colegio no se perciben como tales y por lo general son tomadas como "juego". Las más frecuentes son los apodos que hacen alusión a las características físicas de los jóvenes y que son atribuidos entre ellos. La evidencia de que el colegio es una mixtura, relativa, de procedencias sociales da pie a la idea de que el centro educativo es una mezcla de clases y de culturas que convive sin conflicto.

Profesores y directivos no niegan que se den actitudes racistas en los colegios; aceptan que estas sí están presentes, sobre todo en los primeros años, en el nivel primaria, en donde los niños discriminan por la pertenencia de accesorios electrónicos y ropa de marca. En este nivel, se va trabajando en la aceptación del Otro a lo largo de la experiencia escolar, de modo tal que cuando llegan a secundaria, esto ya no se presenta. Señalan, sin embargo, que la influencia mayor viene de las familias, las cuales buscan diferenciarse formando grupos cerrados. En ese sentido, el colegio va reeducando en esta dimensión, en conflicto con la familia: "Cuando son más niños, la tendencia es a rechazar al cholito, al negrito; esto se va trabajando en los grupos, y al terminar segundo de Secundaria, esto está normalmente superado" (entrevista profesor $P$ ).

\section{Rasgos lingüísticos que caracterizan el discurso de los escolares}

En nuestro afán por explorar sobre las percepciones de los estudiantes acerca de ciudadanía y democracia pensamos que era interesante aplicar un análisis lingüístico a las entrevistas de los estudiantes de los colegios. Así, este análisis nos dio pistas sobre la manera en la que los estudiantes hablan sobre su formación en valores ciudadanos complementando el análisis de contenido realizado en la investigación del IED.

En este sentido, el análisis lingüístico fue revelador. Las partes de las transcripciones en las que los estudiantes comparten sus percepciones sobre la formación en valores democráticos presentan las características del lenguaje académico, lo que se traduce en el uso de definiciones, de verbos modales (e.g., poder), de infinitivos y de la voz pasiva.

Asimismo, prevalecen el uso de la tercera persona, del término "alumno" y del rol de beneficiario versus el de actor asumido por los estudiantes (e.g., 'nos enseñan' vs. 'nosotros aprendemos'). La presencia de estos rasgos lingüísticos en el discurso de los estudiantes favorece la impresión que ellos asumen un

110 I rol pasivo con respecto a su formación integral. A continuación explicamos con 
más detalle las características comunes encontradas en las entrevistas de los alumnos:

\section{Similitudes con el lenguaje académico}

Al contestar la pregunta acerca de qué entienden por ciudadanía o por ser ciudadano/as, los estudiantes, tanto de los colegios privados como los de los diferentes Colegios de Fe y Alegría respondieron en forma muy similar. En los siguientes dos extractos se puede apreciar cómo proveen definiciones mediante el uso de infinitivos como tener responsabilidad, sentirse parte del Perú, introducirse al mundo social, influenciar a los más pequeños para el futuro, , acogerse a ella, plantearse soluciones, entre otras ${ }^{15}$.

Para mí [ser ciudadana] es tener responsabilidad para lo que es el Perú... me refiero a que uno cuando cumple la mayoría de edad [inaudible] sentirse parte del Perú... como cuando uno quiere a su país y va a votar lastimosamente es obligatorio porque para mí ir a votar es porque uno quiere a su patria. . . es lo que yo entiendo quiere a su patria. . . introducirse al mundo social adulto... influenciar a los más pequeños para el futuro.... enfrentarse a la vida con más madurez, asumiendo las responsabilidades que vamos cometiendo. . . ser más responsables y tener muchos cambios. (Entrevista con alumnos $F$ ).

[Ser ciudadano] es ser parte de la sociedad acogerse con ella plantearse soluciones para que la sociedad vaya por mejor camino.... . . ser ciudadano es también ser parte del colegio es decir que no solamente es ser mayor de 18 el mismo niño es ciudadano porque él da sus sugerencias y opiniones que también puede hacer cambiar a la sociedad a una mejor. (Entrevista con alumnos $F$ ).

Asimismo, se aprecia el uso de verbos modales como puede y tiene (que):

15 Es importante anotar que en la entrevista con alumnos del Colegio Fe y Alegría, un alumno se expresó con la primera persona del plural "nosotros", rasgo muy poco usado por los estudiantes en todos los colegios para expresar su percepción de qué es ciudadanía. El uso de nosotros da la impresión de que se asume un rol más activo por parte del hablante. 
[Ser ciudadano] puede ser una persona que tiene voz tiene voto y pueda dar sus expresiones pueda comunicarse con la gente y todo eso... siempre tiene que participar ser activo para un mejoramiento de una comunidad. (Entrevista con alumnos F).

Por último, otro elemento común es el uso de la voz pasiva refleja en "se considera ciudadano":

Casi mayormente en la sociedad se considera ciudadano o ciudadana ... a los mayores de 18 años nosotros somos considerados parte de la sociedad pero no como ciudadanos. .. nosotros participamos como ciudadanos en el colegio y segui[mos] con la participación activa fuera del colegio ayudando a que nuestra ciudad progrese. (Entrevista con alumnos F).

El lenguaje académico ha sido objeto de estudio de varios autores (Lemke, 1990; Martin, 1993; Wignell, 1994; Veel, 1997). En su mayor parte, estos estudios se centran en la lengua inglesa. Sin embargo, Gibbons \& Lascar (1998) y Gibbons (1999) también han aplicado la noción de registro al español. Los autores concluyen que al igual que en inglés, en español también se puede hablar de una variedad de registros académicos que son propios del lenguaje usado y promovido en la escuela. Martin (1993), por ejemplo, concluye que el lenguaje de los textos científicos se caracteriza por el empleo de términos técnicos y de formas de razonamiento particulares a la actividad científica. Esto se debe a que los autores tienen como objetivo principal describir, clasificar y explicar los fenómenos de la realidad dado que la tarea de la ciencia es predecir y controlar dichos fenómenos. De esta manera, el lenguaje científico se adapta a las necesidades de expresión que la ciencia demanda. Así, se afirma que el lenguaje de la ciencia es expositivo o analítico en su mayor parte y se usa para expresar clasificaciones, taxonomías y relaciones lógicas entre procesos (Lemke, 1990, p. 158). Otra característica propia del lenguaje científico es la pretensión de la objetividad. La forma en la que se presenta la información es neutral y aparentemente carente de ideología. Es usual que para ello se recurra a expresiones lingüísticas que "filtren" la ideología y aparenten objetividad. Uno de estos recursos es el uso de la voz pasiva, el otro es la atemporalidad en los verbos, ya sea usándolos en presente o en infinitivo. Nos llama la atención que sean estos elementos lingüísticos los más recurrentes en las declaraciones de los alumnos sobre ciudadanía y democracia. 
En resumen, los rasgos del lenguaje académico presente en el discurso de los estudiantes al referirse a su formación en valores democráticos y ciudadanos le da al discurso una apariencia muy formal pero sin contenido. Nos parece que esta sensación de formalidad en el discurso converge con la idea de participación simbólica o decorativa desarrollada anteriormente.

\section{El rol que asumen los estudiantes en su formación integral}

Es muy frecuente encontrar en los enunciados de los estudiantes sobre la formación en valores que asumen un rol de beneficiario en lugar del de agente. Esta distinción se puede comprender fácilmente si comparamos los siguientes ejemplos:

(1) Pedro estudia computación.

(2) A Pedro le enseñan computación.

En la oración (1), el sujeto Pedro realiza la acción, por lo que tiene el rol de agente o actor. En cambio, en la oración (2) la acción recae sobre Pedro; el rol es el de beneficiario. En forma similar hallamos frecuentemente en las entrevistas de los estudiantes el rol de beneficiario (marcado en negritas) referido a los alumnos y no el de agente (subrayado):

La formación cristiana que nos dan la formación en valores a socializar. . . en las horas de tutorías que tenemos los tutores con los alumnos llevan a cabo charlas reuniones y llevan temas buenos para los jóvenes problemas de Tacna [como] las drogas los embarazos precoces en las chicas los vicios de Internet el acceso a pornografía... . . los profesores nos hablan nos aconsejan y nos orientan diciendo que estos temas no son correctos. (Entrevista alumnos F).

$P$ : ¿Cómo hace el colegio para formar estos valores?

$R$ : Asumiendo nuestros actos con responsabilidad que lo que haqamos mal nos demos cuenta y no volverlo a cometer. . . son enseñanzas que vienen del hogar y son puestos en práctica en el colegio con las tareas, el respeto a los profesores, ser sincero. (Entrevista con alumnos F).

Nos enseñan a ser veraces con nosotros mismos dejándonos esa responsabilidad a nosotros. . . por ejemplo en religión nos dan ciertos momentos a la que reflexionemos nos dejan trabajos a casa. . . siempre nos dan 
consejos y hablamos sobre lo que queremos lograr. . nos dan a entender que nosotros podemos elegir lo que queremos... nos ayudan a comprender que parte de las enseñanzas nosotros podemos desarrollarnos aun más nuestras capacidades dan más. . . con los padres con la familia y con nosotros mismos porque si no parte de nosotros no vamos a lograr nada. (Entrevista con alumnos F).

Para mí formarse como personas, sería en todos los aspectos y en todas las dimensiones que podría estar en el hombre, en el campo de la religión, acá hacen eso, por lo menos nos invitan a confesarnos, hacemos misa en cualquier día de la semana no seguido, pero por lo menos nos dan la oportunidad de hacerlo al mes, realizar jornadas de algo sirven, charlas, orientaciones. En tutoría nos inculcan temas de los valores y más que todo ponerlos en práctica. . (Entrevista con alumnos $P$ ).

Nos parece muy elocuente el modo como los estudiantes se refieren a su formación en valores: el colegio o los profesores los forman, les dice qué es correcto y qué no lo es. Encontramos muy pocos instantes en el que se ejercen el rol de agente como se aprecia en los dos últimos ejemplos. Curiosamente la percepción de los escolares de los colegios particulares es que el colegio sí fomenta entre ellos "el espíritu crítico", por ejemplo, porque pueden tomar decisiones de "manera autónoma"; sin embargo, al analizar su discurso este es muy similar en la forma a los extractos presentados de los escolares de Fe y Alegría de lo cual vemos que existiría una contradicción entre cómo perciben los escolares su formación y cómo se expresan acerca de ella, es decir, hay un desencuentro entre lo que dicen y cómo lo dicen.

\section{Contraste con otros extractos de las entrevistas con los estudiantes}

La forma de organización del texto y los roles asumidos por los estudiantes en las porciones de las transcripciones en las que narran sus planes de trabajo contrastan con los anteriores. La frecuencia del rol agente aumenta, a través del uso de la primera persona, ya sea en plural (nosotros) o en singular (yo).

El primer trimestre hicimos una mazamorrada el objetivo era identificar el delegado de cada área deporte religión... se les dio una identificación que tiene que ser entregada a fin de año al auxiliar si se pierde tiene que ser pagado... el segundo trimestre estamos haciendo una fiesta para recaudar 
fondos para aumentar y pintar los tachos y el mantenimiento de las plantas... el tercer trimestre estamos haciendo [inaudible] por cada salón para que mantengan su área limpia supervisada por el director auxiliar (...) . . cada uno de nosotros nos descuidamos en un momento lo que consumimos esa basura se nos cae por ahí. . . a veces cuando estás en un lugar muy lejano del tacho te da flojera a algunas personas. . Ios delegados nos han dicho que es un problema, por eso vamos a aumentar tachos. (Entrevista con alumnos F).

Nosotros queríamos tener un plan de actividades para todas las promociones porque si bien hay actividades que siempre se realizan hay otras que siempre se dejan de lado... nosotros pensábamos poner presión sobre los tutores para poder hacer esto. .. queríamos realizar conversatorios los viernes a la hora de salida o tutoría para que los alumnos plantearan sus necesidades ante el tutor porque el tutor normalmente habla y habla pero casi nunca hablan los alumnos. . . tenemos planes de poner una empresa X para sacar más fondos para el consejo para tener a oportunidad de ayudar a quien lo necesite dentro de colegio para compañeros becados. (Entrevista con alumnos $P$ ).

Nos parece significativo encontrar muchos más casos del uso del rol de agente cuando los alumnos se refieren a sus planes de trabajo que cuando hacen referencia a su formación en valores. Pensamos que a través de este uso los escolares reflejan su rol como líderes estudiantiles señalando la autoría de sus planes de trabajo.

En conclusión, el análisis lingüístico revela que los estudiantes asumen un rol pasivo al referirse a su formación ciudadana y democrática. Nos parece elocuente que los estudiantes demuestren un rol más bien activo en otras partes de sus discursos.

\section{Ausencias en el discurso de los escolares}

Nos pareció importante incluir en nuestro análisis lo que no encontramos en el discurso de los estudiantes. Por ejemplo, en ninguna de las entrevistas vemos al menos una demanda de los escolares con respecto a su formación en valores de los colegios. Por el contrario, la impresión que nos dejan es que están totalmente identificados con sus colegios. Cabe recordar que las entrevistas se realizaron con los líderes estudiantiles, quienes son los representantes de los escolares en cada colegio. En ese sentido nos llama la atención que no halláramos indicios 
de cuestionamientos por parte de los líderes estudiantiles con respecto a su formación en valores. La falta de sentido crítico a la formación en valores nos parece significativa, en tanto que la dimensión pedagógica de la educación popular "busca desarrollar una pedagogía que fomente el espíritu crítico, creativo e innovador que el reto de transformar la realidad requiere" (Fe y Alegría, 2005, p. 12).

Asimismo, en los Colegios de Fe y Alegría no encontramos ningún comentario, ni siquiera sutil, que pudiera indicar cierta incomodidad sobre algún aspecto de su vida escolar. En cambio, en los colegios particulares hallamos algunos instantes en los que los escolares se atreven a mostrar su descontento. Pensamos que valdría la pena profundizar en este aspecto en una investigación posterior, por ejemplo, afinando las técnicas de acopio de información.

Otro vacío notable sale a relucir cuando los escolares de los colegios particulares hablan sobre su futuro. En ninguna de estas entrevistas hallamos referencias a trabajar. Por el contrario, los estudiantes de los colegios particulares proyectan su futuro inmediato en estudiar para ser profesionales; es más, muchos de ellos ya habían sido aceptados en la universidad, otros estaban en academias pre-universitarias. En contraste, la proyección de futuro inmediato de los estudiantes de Fe y Alegría es trabajar, para ayudar a su familia, y estudiar ${ }^{16}$. Incluso se menciona al trabajo como medio para alcanzar los estudios superiores.

\section{Conclusiones}

En este artículo nos propusimos explorar las percepciones de los estudiantes de secundaria acerca de ciudadanía y democracia, a partir de su experiencia escolar, comparando la producción de sus discursos de acuerdo con sus respectivos espacios escolares. Los dos métodos de análisis revelan coincidencias entre los discursos de los estudiantes de los colegios jesuitas particulares y los de los colegios de Fe y Alegría.

Debido a la naturaleza exploratoria de nuestro estudio, más que conclu-

16 La población escolar de ambos tipos de colegios tiene características socio económicas diferentes; los estudiantes de los colegios particulares provienen predominantemente de familias de sectores medios y altos urbanos, mientras que los escolares de los colegios de Fe y Alegría provienen de familias pobres, urbanas y rurales. En el estudio trabajamos solo con los colegios localizados en centros urbanos; sin embargo, hay que considerar que los colegios Fe y Alegría de Arequipa y Tacna tienen también escolares migrantes de primera generación de origen rural. 
siones proponemos futuras líneas de investigación en relación a: a) las fuentes institucionales usadas por los colegios que remiten a los fundamentos de la formación ciudadana; b) la participación en la vida escolar y la formación de la ciudadanía; c) las características lingüísticas del discurso de los estudiantes respecto a su formación en ciudadanía y democracia; d) las percepciones de los escolares en cuanto al trabajo; y e) las opiniones de los estudiantes acerca de su formación integral.

\section{Las fuentes institucionales de la formación de la ciudadanía}

Tanto los colegios de la Red Fe y Alegría como los colegios jesuitas particulares cuentan con un corpus documental institucional que constituye el referente de su labor formativa. Algunos elementos contenidos en estos documentos que se relacionan con el problema de estudio son la educación personalizada, la participación crítico reflexiva, la formación en valores orientadores del comportamiento actual y futuro. La presencia efectiva de estos referentes en la formulación y ejecución de los programas curriculares es aparentemente muy distinta en los colegios Fe y Alegría y en los colegios jesuitas particulares. En los primeros, la Propuesta Pedagógica es un referente inmediato y conocido por el cuerpo directivo y docente de los colegios; y busca ser traducido en los programas curriculares de los centros educativos. En los segundos, son referentes más mediatos y su conocimiento está limitado generalmente a los directores y a algunos directivos.

En ambos casos cabría indagar si estos "textos fundamentales" alimentan efectivamente las prácticas educativas y cómo lo hacen; si estos son materia de revisión y reflexión para actualizar sus contenidos; o, por el contrario, quedan como una suerte de códigos finalmente poco actuantes sobre las prácticas educativas.

Los colegios Fe y Alegría tienen otras fuentes inspiradoras de su labor formativa, dadas por el carisma de la congregación religiosa que regenta el colegio; algunos reconocen, además, la "espiritualidad ignaciana" como un referente de la propuesta formativa. Cabría profundizar en el conocimiento de la manera en que estas diferentes fuentes se interrelacionan, aportan contenidos específicos, influyendo en la concepción de la formación de la ciudadanía (qué y cómo) de las personas a cargo de la dirección del colegio.

Los colegios jesuitas particulares se encuentran en un proceso de construcción de un proyecto pedagógico común; en dicho proceso cabría identificar los 
diferentes entendimientos sobre la formación de la ciudadanía y cómo estos se traducen en el diseño curricular. El supuesto que encontramos es que hay en el cuerpo docente y directivo diferentes concepciones de lo que significa formar la (o para la) ciudadanía pero estas no son siempre explícitas; se asume que existe un entendimiento común al respecto.

\section{La participación en la vida escolar y la formación de la ciudadanía}

Los colegios otorgan una gran importancia a la promoción de la participación activa de los y las estudiantes en la vida escolar; la participación en sí misma sería una expresión de disposiciones, actitudes y valores propios de la democracia y del ejercicio ciudadano. Los colegios alientan esta participación -algunas veces estableciendo criterios de evaluación escolar vinculados con ella-; facilitan la creación de comisiones y grupos de trabajo de profesores y alumnos, además de promover la elección de representantes estudiantiles con mecanismos propios de la democracia (competencia, deliberación y voto universal). La existencia de consejos estudiantiles y de municipios escolares elegidos por los propios estudiantes, es vista en sí misma como una escuela formadora de valores propios de la ciudadanía democrática. No obstante, hemos identificado que los contenidos de dicha participación no necesariamente estarían siendo formadores de "virtudes democráticas", tales como la expresión y el ejercicio de la representación de intereses estudiantiles, la capacidad de actuar concertadamente para lograr propósitos comunes, y la responsabilidad frente a los electores mediante mecanismos de rendición de cuentas.

De manera particular, en los colegios Fe y Alegría, la participación estudiantil parece ser más funcional a los intereses y necesidades definidos por la institución educativa -tales como el control del orden, la disciplina y la limpieza- que a la expresión de intereses propiamente estudiantiles como, por ejemplo, intereses recreativos y culturales, orientación vocacional y de la vida afectiva, o mayor desarrollo de competencias para la inserción en el mundo laboral.

Profundizar en ello requeriría mirar con mayor detenimiento los planes de trabajo de los consejos estudiantiles; la relación representantes-autoridad escolar, sobre todo el papel de los profesores asesores que son mediadores en esta relación; los roles asignados a los consejos estudiantiles y, en general, el 
de los colegios, así como en el imaginario de los escolares (no solamente de los líderes estudiantiles); así como los mecanismos de relación representantesrepresentados.

\section{Los rasgos lingüísticos que caracterizan el discurso de los escolares}

El uso de rasgos similares a los del lenguaje académico al referirse a los conceptos de ciudadanía y democracia, de un lado, y la evidencia de lo entienden por ciudadanía y democracia, de otro, se conjugan para dar la impresión que el discurso de los escolares se queda en un nivel formal y daría cuenta de un aprendizaje más teórico que de contenido vivencial porque no atraviesa su identidad escolar-juvenil. Sería interesante poder recoger más información para profundizar en este aspecto teniendo en cuenta el análisis lingüístico como parte del diseño del estudio y no posterior al mismo.

\section{Contrastes entre las percepciones de los estudiantes respecto al trabajo}

Las declaraciones de los escolares de Fe y Alegría en cuanto a sus perspectivas para el futuro revelan que tienen muy presente el trabajo como medio para lograr sus metas. Por el contrario, los escolares de los centros particulares no hacen ninguna mención a trabajar en el futuro, solo se proyectan como estudiantes. Cabe recordar que el valor del trabajo está contenido en la Propuesta Pedagógica de Fe y Alegría como parte de la formación escolar. Este contraste entre los estudiantes de colegios particulares y los de Fe y Alegría nos lleva a preguntarnos en qué medida la formación en valores en el colegio podría tener un impacto en destacar el trabajo como valor entre los escolares de los colegios particulares o si es este más bien viene determinado por la condición socioeconómica de los alumnos.

\section{Ausencia de cuestionamientos por parte de los escolares a su formación}

En las declaraciones de los escolares no hallamos opiniones críticas sobre su formación integral. Ello sumado a los resultados que arroja el análisis lingüístico de sus opiniones nos daría la impresión de que, en general, los estudiantes asumen un rol pasivo en cuanto a su formación en valores. Dado a que en los 
colegios particulares como en los de Fe y Alegría existe un énfasis por desarrollar el "espíritu crítico", nos parece relevante indagar más en este aspecto afinando la metodología de investigación en futuras investigaciones.

\section{Referencias bibliográficas}

Alfageme, E., Cantos R. \& Martínez M. (2003). De la participación al protagonismo infantil. Propuestas para la acción. Madrid: Plataforma de Organizaciones de Infancia.

Benavides, M.; Cueto, S. \& Villarán, V. (1999). Socialización escolar y educación en valores democráticos: el caso de las escuelas alternativas. En A. Panchifi \& M. Valcárcel (Eds.), Juventud: sociedad y cultura (pp. 133-170). Lima: PUCP, Universidad el Pacífico, IEP.

Caijao, F. (1998). Educación ciudadana y democratización de la escuela. En P. Arregui \& S. Cueto (Eds.) Educación, ciudadanía, democracia y Participación (pp. 142-153). Lima: GRADE.

Carbajo, J.L. \& Espino, G. (2001). Estudiantes y Ciudadanos. Líderes escolares en Ayacucho. Lima: TAREA.

Compañía de Jesús Provincia del Perú. (2002). Lineamientos para el Proyecto Educativo Común de los Colegios Jesuitas del Perú. Lima: Compañía de Jesús Provincia del Perú.

Conferencia de Provinciales Jesuitas de América Latina. (2005). Proyecto Educativo Común de la Compañía de Jesús en América Latina. Río de Janeiro: CPAL.

Eguren, M. (2006). Educación y participación: enfoques y prácticas promovidos desde el Estado, la sociedad civil y las escuelas. En C. Montero (Ed.), Escuela y participación en el Perú. Temas y dilemas (pp. 43-127). Lima: IEP.

Epstein, E. (1998). Dislocamiento cultural por acción del Estado: la paradoja de la educación democrática. En P. Arregui \& S. Cueto, (Eds.), Educación, ciudadanía, democracia y participación (pp. 110-124). Lima: GRADE.

Fe y Alegría. (2005). Propuesta Pedagógica. Lima: Fe y Alegría del Perú.

Fernandez, G. (2001). La ciudadanía en el marco de las políticas educativas. Revista Iberoamericana de Educación, 26, 167-199.

Gibbons, J. (1999). Register aspects of Literacy in Spanish. Written language and literacy, 2 (1), 63-88. 
Gibbons, J. \& Lascar, E. (1998). Operationalising Academic Language Proficiency in Bilingualism Research. Journal of Multilingual and Multicultural Development, 1 (1), 40-50.

Halliday, M. A. K. (1978). Language as social semiotic. Londres: Edward Arnold.

Halliday, M. A. K. (1985). An introduction to functional grammar. Londres: Edward Arnold.

Halliday, M. A. K. (1994). An introduction to functional grammar. Londres: Edward Arnold.

Lemke, J. (1990). Talking science: Language, learning, and values. Norwood, NJ: Ablex.

López, S. (1997). Ciudadanos reales e imaginarios: concepciones, desarrollo y mapas de la ciudadanía en el Perú. Lima: IDS.

Lüchmann, L. H. (2006). Os sentidos e desafios da participação. Ciências Sociais UNISINOS, 42 (1), 19-26.

Máiz, R. (2003). Nacionalismo y Multiculturalismo. En A. Arteta, E. García \& R. Máiz (Eds.), Teoría Política: Poder, Moral y Democracia (pp. 424-462). Madrid: Alianza.

Martin, J.R. (1993). Literacy in science: Learning to handle text as technology.

En M. A. K. Halliday \& J. R. Martin (Eds.), Writing science: Literacy and discursive power (pp. 166-202). Pennsylvania: University of Pittsburgh Press.

Reimers, F. \& Villegas-Reimers, E. (2006). Sobre la calidad de la educación y su sentido democrático. Revista PRELAC, 2, 90-108.

Rodriguez, Y. (2009). La Formación de la Ciudadanía desde la Experiencia escolar. Anhelos y Procesos en los Colegios de la Compañía de Jesús en el Perú. Lima: Universidad Antonio Ruiz de Montoya.

Sabatini, C. (1998). Educación Ciudadana: qué y cómo de las mediciones de impacto. En P. Arregui \& S. Cueto (Eds.), Educación, ciudadanía, democracia y participación (pp. 85-97). Lima: GRADE.

Schleppegrell, M. J. \& Achúgar, M. (2003). Learning language and learning history: A functional linguistics approach. TESOL Journal, 12 (2), 21-27.

Schleppegrell, M. J., Achúgar, M. \& Oteíza, T. (2004). The Grammar of History: Enhancing Content-Based Instruction through a Functional Focus on Language. TESOL Quarterly, 38 (1), 67-93. 
Veel, R. (1997). Learning how to mean-scientifically speaking: Apprenticeship into scientific discourse in the secondary school. En F. Christie \& J. R. Martin (Eds.), Genre and institutions: Social processes in the workplace and school (pp. 161195). Londres: Cassell.

Wignell, P. (1994). Genre across the curriculum. Linguistics and Education, $6(4), 355-372$. 